\title{
Implementation Considerations for Big Data Analytics (BDA): A Benefit Dependency Network Approach
}

\author{
Juane Maritz $\left(\mathbb{0}\right.$, Sunet Eybers ${ }^{(\varpi)}(\mathbb{C}$, and Marie Hattingh $(\mathbb{C}$ \\ University of Pretoria, Private Bag X20, Pretoria 0028, South Africa \\ maritzjuane@gmail.com, \\ \{sunet.eybers, marie.hattingh\}@up.ac.za
}

\begin{abstract}
The benefits of Big Data Analytics (BDA) are substantial in instances where organisations manage to successfully implement analytical capabilities. These benefits include improved, data driven decision-making, which can lead to deeper insight into business operations and as a result better performing organisations. Not surprisingly, an increased number of organisations are researching best implementation practices for BDA projects.

Similar to software projects, research has shown that many BDA projects fail or do not deliver the business value as promised. To address this issue, the main objective of this research is to identify BDA implementation considerations for new BDA endeavors that will help organisations to align their BDA efforts with their overall business strategy to maximize business value.

Based on a Benefit Dependency Network (BDN) model as main theoretical underpinning, a structured literature review was conducted focusing on investment objectives, business benefits, enabling changes and IT enablers when implementing BDA. A BDA implementation requires a holistic approach by considering aspects such as the skills of people which will have an impact on the structure of the organisation, business processes and technology changes to deliver benefits and investment objectives. Each of the domains of the BDN should be considered prior to BDA implementations.

The research offers a guideline to organisations implementing BDA, based on the foundation of BDN.
\end{abstract}

Keywords: Big Data Analytics $\cdot$ Structured literature review $\cdot$ Benefit Dependency Network (BDN)

\section{Introduction}

Data is continuously being created and shared by people, tools and machines [1]. Huge data sets can be attributed to the introduction, evolvement, and advancement of new sharing technologies, the connectivity of devices and number of data generating devices/platforms like Facebook, internet connected cars, wearable devices and cell phones [3]. Big datasets often contain untapped, hidden value. To realise the value of big data, organisations need to apply sophisticated analytical methods to extract actionable 
insight from these datasets. As a result, organisations acknowledge the need for Big Data Analytics (BDA) to help drive decisions making within the organisations [4].

Despite the obvious financial benefits of big data analytics, organisation still face challenges implementing BDA projects [6]. One of the suggestions is that BDA can only be implemented successfully where organisation understand the organisational changes that needs to be considered before embarking to such implementations, and, similar to IT implementations, fully understand the requirements [7].

The need for a holistic BDA implementation guideline lays the foundation for this research. It is argued, for an organisation to successfully implement BDA, the organisation and technical IT teams must work together to successfully implement BDA [8]. An all-encompassing approach is therefore required considering multiple facets within the organisation [9]. The research question of this study is 'What are the changes that an organisation need to make to successfully implement big data analytics?'. The research is therefore conducted through the lens of organisational improvement.

The research paper starts with evaluating the meaning of BDA and subsequently identify the challenges associated with the implementation and realization of business value. The Benefit Dependency Diagram (BDN) is explained followed by the research approach. As part of the discussion of findings, a preliminary BDN summarizes findings using the different domains of the BDN where after the research is concluded.

\section{Big Data Analytics (BDA)}

Big data analytics (BDA) incorporates techniques and technologies that are used to capture, store, transfer, analyse and visualize enormous amounts of structured and unstructured data [10]. A good descriptive definition of BDA was proposed by the Operations Research and Management Science stating that BDA is a process of transforming data into insight that will guide business decision making and organisational strategic direction based on insight derived from data to predict the future [11]. BDA therefore includes skills, technologies, methodologies, and practices to analyze data, enabling organisations to better understand their customers, their markets and to help drive future decision making [12].

According to the Chartered Global Management Accountant (CGMA), (a governing body focusing on governing accountants), BDA constitutes four levels namely reporting, analysis, monitoring and prediction [13]. Reporting visualizes data and can explain past events within the organisation. Analysis refers to the deep understanding of possible reasons for past events. Monitoring enables the organisation to understand what is currently happening within the organisation. Prediction is the action of using data and determining what could happen in future within the organisation, i.e. predict future events [13]. Although BDA can assist with providing insight on all four levels, the strength of BDA lies in the ability to predict and anticipate events based on current data. As a result, BDA can assist decision makings to create appropriate business strategies, providing them with the right knowledge to the correct people at the right time and in the right format [14]. Only after performing data analytics tasks can the 
organisation gain insight from big data. Insight is an actionable piece of knowledge that can drive decision making, strategic decision making and improve productivity and efficiency [2].

\subsection{BDA Implementation Challenges}

Although BDA promise a lot of advantages to the business, research has indicated that managers and organisations struggle with the successful implementation of Big Data Analytics strategies, in particular aligning data analytics projects with current business strategy [13, 15]. Furthermore, research has indicated that management does not comprehend how to identify business benefits from BDA implementations [16].

The reason why organisations struggle to realise business value from BDA can be grouped into three main categories, namely technical related challenges, managerial challenges as well as organisational challenges. Technical challenges include data related issues (challenges consolidating data from different sources and data quality); challenges with the presentation of findings (intelligent visualization and presentation of data) and making the appropriate technology choices [4, 13]. Managerial challenges include the inability of managers to gain insight from data analytics results, and subsequently linking the insight to actual business problems with the objective of increasing revenue [13]. Organisational related challenges include changes in decision making and leadership, organisation culture changes, adoption of a data-driven decision-making culture and clear direction of initiatives [10].

\subsection{Realising the Value of BDA}

The challenge to implement new IT technologies, such as BDA, has been ongoing for many years. An astonishing $70 \%$ of most IT projects fail or deliver the actual benefit promised when implementing the new technology [17]. Research has shown that IT projects fails due to numerous reasons, of which change management, both on organisation as well as process level, are the most prevalent [18]. According to Peppard [17], benefits from IT investments don't just "happen" - it needs a firm commitment from organisations to drive the investment through organisational change [17].

Multiple proprietary tools have been developed to assist the organisation in making IT investment decisions. Examples include a SWOT analysis [19], Balanced Scorecard [20] and the Benefit Dependency Network (BDN) diagram [17] (to name a few). These tools consider possible intangible benefits of investments and can, when used properly, assist management to maximize the benefits of their IT investment by conducting a proper evaluation of the planned investment. These tools are also designed to assist organisations in the alignment of their IT investments to the overall business strategy.

The BDN was selected as main theoretical foundation for this study. The tool was selected due to the powerful visual ability to graphically highlight change requirements, both on a holistic (enterprise) and functional level, that managers can use as a guide to evaluate the extent of changes required prior to embarking on new IT investments, in this instance the adoption of BDA [17]. It furthermore considers the relationship between people, processes, and technology assisting managers to understand how the expected benefits will be delivered through the combination of technology and business 
changes. From an organisational perspective the one-page graphical model assists with the alignment of IT investments with the business strategy and highlights the fact that IT investments should not be driven by technology but rather by clear business objectives.

The BDN is not a widely adopted and researched tool and does not consider the strengths, weaknesses, constraints and challenges that organisations might face when adopting new technologies. Although critics feel that the model lacks strong empirical evidence, the tool seemed appropriate for this level of initial investigation into the identification of organisational adjustments required prior to implementing BDA. Furthermore, it was only used as a lens to analyse data and guide the discussion.

\section{Research Approach}

The main purpose of this research was to identify strategic level implementation considerations prior to the implementation of BDA. As a result, the research aimed to identify how the maximum value can be achieved from BDA implementations.

A structured literature review was conducted, using keywords such as big data implementation, big data analytics implementation, data analytics implementation, analytics implementation model, business intelligence and implementation. Business Intelligence (BI) was included as a search term because BI was perceived as part of a BDA implementation. Peer reviewed articles were only considered when written in English, for the period 2015 to 2019, on the following databases: ABI/Inform collection, EBSCOhost and Emerald Insight. Both the Forbes and Harvard Business Review online sources were consulted. There was no restriction placed on the discipline on which the article focused as IT is perceived as a multidisciplinary field.

The initial, preliminary number of articles returned after the search process was 2453. Following the application of the inclusion criteria it was reduced to 300 . This preliminary pool of 300 articles were further evaluated for applicability by considering the abstract section of each article using the proposed quality assurance criteria by Kitchenham and Charters [21]. The criteria allowed a score rating of $0,0.5$ or 1 to be allocated to each article based on a clear description of the research process, identification of clear research limitations and finally the extent to which the research was applied in practice. The final article pool consisted of 15 articles.

Each of the 15 top articles were evaluated and classified by means of thematic content analysis using each of the 'domains' of the BDN tool (namely the investment objectives, business benefits, business changes, enabling changes and IT enablers, as described in Sect. 2.2). A matrix was created listing the each of the domains horizontally with a specific measurement identified in literature supporting the domain. For example, articles indicating a clear statement of what the BDA implementation should achieve was included in the investment objective; advantages and benefits achieved as part of a BDA implementation was included under the business benefits section; permanent business changes such as business processes as part of the business changes heading; enabling changes referring to non-permanent, short term changes in the organisation; and IT enablers refers to any technology related requirements for BDA. Table 1 contains an example of the matrix used. The authors of the various articles 
were listed on each table row. Where research focused on a particular concept, an X was used in the table to indicate research applicability.

Table 1. Concept mapping matrix

\begin{tabular}{l|l|l|l|l|l}
\hline \multicolumn{1}{l}{ Concept } & $\begin{array}{l}\text { Investment } \\
\text { objectives }\end{array}$ & $\begin{array}{l}\text { Business } \\
\text { benefits }\end{array}$ & $\begin{array}{l}\text { Business } \\
\text { changes }\end{array}$ & Enabling changes & IT enablers \\
\cline { 2 - 7 } & $\begin{array}{l}\text { What BDA } \\
\text { implementation } \\
\text { objectives }\end{array}$ & $\begin{array}{l}\text { Organisational } \\
\text { advantage and } \\
\text { benefits }\end{array}$ & $\begin{array}{l}\text { Permanent, } \\
\text { long term } \\
\text { organisational } \\
\text { change }\end{array}$ & $\begin{array}{l}\text { Non-permanent, } \\
\text { short term } \\
\text { organisational } \\
\text { change }\end{array}$ & $\begin{array}{l}\text { Technology } \\
\text { requirements }\end{array}$ \\
\hline Author & & & & & \\
\hline
\end{tabular}

\section{Discussion of Findings}

The discussion of findings is based on the summary of the final pool of 15 articles (available on request). As mentioned previously the analysis was conducted per domain, starting with the investment objectives, business benefits, business changes enabling changes and IT enablers (read from right to left on the BDN). The order of the domains is of extreme importance to ensure that technology support the overall investment and align with the business strategy. Arrows on the diagram indicate a relationship or inter-domain dependency between. A change in one of the items can influence another domain and can therefore assist in the identification of change to be consider imperative to realising benefits (see Fig. 1).

Any benefits management intervention starts with a clear need to change, in this instance referred to as the "driver". Drivers are elements that top management regard as the main driving force behind the need for the business to change [22, 23], and can be both internal and/or external to the organisation. In this instance, the organisation wants to adopt BDA due to the need to analyze huge amounts of data. For example, they need to replace existing BI systems and move towards the implementation of BDA which will lead to improved sales and customer service. BDA is therefore the driver or reason behind the planned intervention [22].

\subsection{Investment Objectives}

Investment objectives are specific to the project and focuses on the outcome of the project, i.e. what the project will achieve if successful [22]. It is set for a particular period (linked to the project duration) and are specific, measurable, achievable [24].

Fourteen (out of fifteen) articles evaluated indicated that they identified reasons for investing in BDA prior to the actual investments made (for example [8, 12, 29]). Ten articles agreed that organisations invest in BDA with the main objective to achieve business transformation (for example [8]). Multiple definitions existed for business transformation, but the trend was clear that business transformation happens through the identification of new business models, products and services that are identified 
through BDA activities. BDA leads to fundamentally changing processes, people, technology and organisational structure across the organisation that lead to business transformation. No clear consensus was reached on what business transformation is, but all agreed it can be achieved through the introduction of new/refined products, services and new technology through BDA activities.

Organisations also invest in BDA to improve decision making. Nine articles indicated that most organisations base their decisions on the intuition and experience of employees within the organisation $[12,29]$. This is a flawed process as optimal decision making can only be made based on timeous information [25, 26]. Big Data Analytics enables organisation to integrate various data sources and make various data sources available to the employees for improved decision making [8, 29]. Unfortunately, employees could therefore only improve decision making if they have access to BDA. Therefore, a clear dependency exists between data driven decision making and big data analytics system availability and adoption.

An analysis of the articles further indicated that BDA had the potential of identifying flaws in business processes. BDA can also be used as a risk mitigation tool by means of the following: identifying data leakage, poor performance in business processes, lack of adhering to processes, and gaps in processes [27]. As a result, BDA can assist organisations in the implementation of better business processes by removing flawed business processes to increase efficiency. More efficient processes reduce risks and can assist in achieving the organisational objective of fraud prevention [2].

The overarching trends identified as the main reasons for investing in BDA was to gain competitive advantage through the attraction of more customers, increasing organisation performance, timeous and improved decision making and decreased product time to market.

Only one article explicitly stated the need to increase revenue as an important investment objective of BDA [27]. The results of the remainder of the articles indicated an indirect relationship to increases revenue. An interdependency therefore exists between the need to increase revenue and all other investment objectives. For example, when improving process efficiency revenue will increase by reducing wasted cost. The need for increased revenue is therefore an important investment objective to create business value.

\subsection{Benefits}

Benefits are advantages that is incurred as a result of the project and can be financially related. The investment objectives can only be achieved if some business benefits are incurred [22]. When these benefits are delivered, they will lead to achieving the investment objectives. Thus, to achieve objectives benefits must be delivered. The identified business benefits can serve as Key Performance Indicators (KPI's) by top management to ensure that progress of the projects. This is also used to determine if BDA projects are still aligning to project roadmap [24].

Many overarching business benefits were identified through the analysis of the articles. As a result, it is anticipated that the investment in BDA will result in multiple business benefits. Some of these business benefits are discussed below. 
BDA will enable organisations to have a deeper understanding of their customer's needs and behavior, assisting the organisation to reduce their product time to market. This will also enable the organisation to refine their products, services and business processes based on trends identified in the data. By addressing the needs of the customers, it should be easier to retain customers [24].

Another benefit of investing in BDA is the access to refined, processed data that will improve decision making [2, 7]. BDA implementations often provide users with a consolidated view of data from multiple data sources [29]. In some instances, these data sources are available to end users for the first time ever. Employees, on multiple levels of the organisation, are given access to the data, on which decisions could be made.

Based on business process related data and the subsequent analysis thereof, flaws can be identified in processes and improved, contributing to more efficient organisations and increased organisational performance. It is also an important component in the proactive identification of fraud [2].

Organisations will be able to monitor business processes internally as well as external to the organisation. This assist the organisation in the monitoring of competitor markets and competitor trends. Evidence based decision making can be applied to identify new business models based on these trends [2].

BDA furthermore assist organisations in becoming a learning organisation. A learning organisation imply the ability of the organisation to use analyzed data to transform, adapt and improve the way they function [9].

\subsection{Business Changes}

Business changes are required for an organisation to achieve benefits. It refers to permanent changes to practices, processes and relationships within the organisation to deliver business benefits [28]. These changes cannot normally be implemented until all the enabling changes have been realised. An example of a business change is a an organisational culture change [12].

On a high level, organisational changes focus on data related adjustments as well as organisational environmental changes implemented through proper change management procedures (such as organisation structure, culture and risk management) [8,9].

Organisational structure refers to structural changes an organisation needs to implement prior to BDA. One main theme that was prevalent was multiple aspects of data related adjustments $[8,29]$. For example, structural changes in the way they deal with data needs to be considered. Data is considered to be one of the most valuable assets within organisations. Not surprisingly many business changes evolve around data.

The majority of the articles focused on the need for organisations to implement structural organisation changes ensuring that the data used for BDA is of good quality. The need to have good quality data was not classified as part of the IT enabling domain as it focused on the business process of gathering data (and not on the technology used to gather and store data). Examples include data quality procedures, data access procedures and data security procedures [29].

Good quality data is characterized by integrity (the data is what it says it is), confidentiality (not widely available), reliability (reputable source of data), timeliness 
(recent data), accessibility (controlled access to data), accuracy (level of correctness of data) and completeness (having all sources). Data quality was supported by almost all articles and is also highlighted as the most important business change (for example see [29]).

Another business change that was identified was the relationship between the quality of data and BDA end user utilization [8, 12, 29]. BDA systems will only be used when end users trust the data. End user utilisation is a critical success factor for BDA.

Setting up governance procedures for BDA will create trusted environments for data processing and storage [8, 29]. Trusted environments are environments that has high quality data with high availability. If the systems fail and lose data the organisation loses assets. When losing assets, the organisation lose business value.

Finally, data as an organisational asset needs to be protected. Clear data access processes, rules and access procedures are required specifying user access [29].

BDA requires new skills and new employees therefore require organisational structural changes to the organisational outline such as the hierarchy [8, 12, 29]. For example, new positions will have to be created after the required skills were identified. This will trigger recruitment drives as well as educational and training programs to reskills employees. New risk management procedures will have to be considered to cater for the new organisational outline to protect information and intellectual property. It is important for organisations to implement an effective change management process to assist employees in transitioning into a new organisational structure $[8,9,18]$.

Another major trend that was recognized is the importance of business involvement (i.e. the correct people) in new BDA implementations [27]. Business involvement can be increased by, (a) setting up effective communication channels with decision makers; (b) training of decision makers in the concept of data driven decision making; (c) easy to use systems; (d) linking current business problems to the analysis of data; (e) integration of BDA into daily operations.

\subsection{Enabling Changes}

Enabling changes are adjustments or changes that needs to be implemented for business changes to take place [28]. Examples of enabling changes include defining and agreeing to new work practices, redesigning processes, agreeing on changes to job roles or introducing new job roles and responsibilities, introducing new performance management systems and training and education (for example see [8]). These types of changes can be made before the implementation of the new system.

Similar to the business changes required, data related themes have been identified and considered to be of importance. Other themes identified include human resources related changes, business processes and organisational environmental adjustments [18].

To implement BDA successfully the organisation will have to setup training programs to re-skill existing human resources. A once off, organisation readiness assessment can identify specific skills required prior to the BDA implementation [27]. For example, an organisational readiness assessment can identify new roles such as data scientist and developers with specific analytical skills. The organisation have to 
make provision for these roles in the organisational structure and these roles needs to be defined. A skills matrix can further assist the organisation in establishing the current level of competency of human resources.

The organisation will have to define a number of new processes within the organisation for example support and data related process [8, 29]. The first new process that will have to be established is a data governance process. Data governance processes help to promote data quality and data safety and should be aligned to legislation. It is important that data access roles (i.e. who will be able to view/edit data) is established and included in the data governance procedures. New data security policies and procedures will ensure the safety of data. Support processes will focus on the level of support end users and technical staff members can expect from the business owners. Therefore, new communication channels will be established.

New risk management processes and procedures needs to be implemented to ensure that the data is not compromised [8,9]. Data is one of the organisations most valuable assets as well as the data analysis techniques and findings. The organisation needs to implement procedures to prevent the data assets to be compromised. At this stage, different technological options might be considered.

\subsection{IT Enablers}

IT Enablers are the IT tools that must be implemented as well as IT considerations to be evaluated before introducing a new technology. These items enable organisational change. The majority of articles indicated that Big Data technologies such as Apache Hadoop, NoSQL Data bases such as MongoDB, Apache Cassandra, Apache Mahout, Spark and Storm, are considered [3, 5, 8, 12].

BDA requires data to be consolidated from various data sources. The organisation needs to identify which sources they want to use for their BDA projects and how these will be integrated. Big Data Sources identified in the articles were internal and external databases, clickstream, social media as well as user-generated data [8, 29].

Information needs to be extracted from the identified data sources, for which data extraction procedures will have to be compiled as well as the appropriate technological tool selected [2, 9]. Analytical tools will also be considered at this stage as the extraction and analytical tool is often bundled together.

Big Data requires scalable storage solutions to enable large amounts of data processing. The organisation also need to keep in mind that massive amounts of data will be entering the organisation. The organisation need to look at their network infrastructure to ensure that the infrastructure will support increased data volumes [8].

The organisation need to consider various visualization tools and platforms to display data analytics [3]. These tools should be user friendly with high availability and acceptable response times [8] as this can increase the adoption rate of BDA.

Rapid scaling cloud platforms are available for organisations to deploy their BDA solutions. This means that organisations can outsource their BDA solutions and therefore do not need to take responsibility for the maintenance of servers and high amounts of data flowing into their solutions. The organisation should, however, consider regulations when storing information on cloud platforms and make use of reputable service providers [8, 29]. 


\subsection{Proposed BDN Model for BDA}

Based on the discussion of findings in Sects. 4.1 to 4.5 above, a preliminary BDN model for BDN were created. It provides the reader with a summary of the sections for each of the domains and displays the interrelationships between items, a distinguishing factor offered by the BDN approach.

A typical example of how the BDN could be used: the first identified Investment Objective was to reduce response time to market changes. A clear business benefit of this overall objective will be that emerging customer trends will be identified. This can only be achieved if (new) data sources, containing relevant data, is available to decision makers when required. For this reason, the relationship between system performance ("review system performance", enabling changes domain) are of vital importance and should be considered when selecting data sources. This can only be achieved through the implementation of rapid scaling technology (IT enablers' domain).

During the analysis of the findings, similar elements were consolidated and combined. Data quality was combined with data integrity, confidentiality and accountability [29]. Data integrity, confidentiality and accountability are considered to be characteristics of quality data. Trustworthiness of data and the completeness of data [8, 12, 29], forms part of the characteristics of good quality data and were included as such.

Below is the proposed BDN model constructed from the articles.

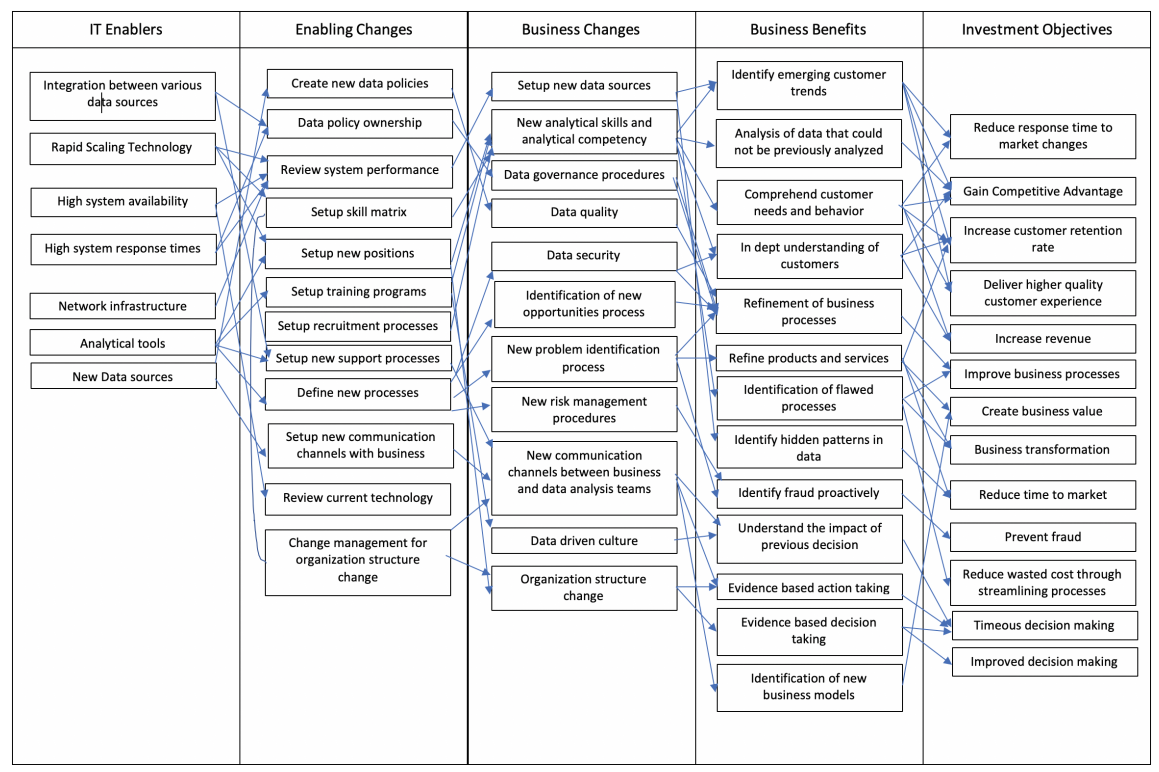

Fig. 1. The BDN analysis of literature 


\section{Conclusion}

The main research question was 'What are the changes an organisation need to consider to successfully implement big data analytics?'. After a systematic structured literature review, a preliminary BDN model was constructed considering the various aspects of the BDN network, namely investment objectives, business benefits, business and enabling changes as well as IT enablers. The model furthermore identified specific relationships between components and the alignment to overall business strategy.

From the results it is clear that BDA implementations should focus on more than just technology. Organisations will have to take a holistic approach when implementing BDA. Considerations should include important decisions with regards to the skills of people, which will have an impact on the structure of the organisation, business processes and technology changes to deliver benefits and investment objectives. Each of the domains of the BDN should be considered prior to BDA implementations.

Finally, BDA is a disruptive technology. Organisations will therefore have to find new ways to implement new technology without completely relying on existing technology. A BDN model approach can assist in identify organisational technology considerations. Further research is proposed to refine the findings of this work.

\section{References}

1. Ernst and Young: Big data - Changing the way businesses compete and operate. EY (2014)

2. McKinsey \& Company: Big Data: The Next Frontier for Innovation. Competition and Productivity. McKinsey Global Institute, New York (2011)

3. Comuzzi, M., Patel, A.: How organisations leverage big data: a maturity model. Ind. Manage. Data Syst. 116(8), 1468-1492 (2016)

4. McAfee, A., Brynjolfsson, E.: Big data: the management revolution. Harv. Bus. Rev. 90(10), 60-68 (2012)

5. Chen, H., Chiang, R.H., Storey, V.C.: Business intelligence and analytics: from big data to big impact. MIS Q. 36(4), 1165-1188 (2012)

6. Chen, M., Mao, S., Liu, Y.: Big data: a survey. Mob. Netw. Appl. 19(2), 171-209 (2014)

7. Court, D., Perry, J., McGuire, T., Gordon, J., Spillecke, D.: Smart Analytics: How Marketing Drives Short-Term and Long-Term Growth. McKinsey \& Company, New York (2015)

8. Wadhwani, K., Wang, D.Y.: Big data challenges and solutions. Technical report (2017). https://doi.org/10.13140/rg.2.2.16548.88961

9. Kholkar, D., Tierney, M.: When data isn't enough: how change management can predict the success or failure of your big data implementation, big data quarterly online (2018)

10. Hopkins, M.S., Krusschwitz, N., LaValle, S., Lesser, E., Schockley, R.: Big data, analytics and the patch from insights to value. MIT Sloan Manage. Rev. 52(2), 21-31 (2011)

11. Saltz, J.S.: The need for new processes, methodologies and tools to support big data teams and improve big data project effectiveness, Santa Clara, CA, USA (2015)

12. Verma, S., Bhattacharyya, S.S., Kumar, S.: An extension of the technology acceptance model in the big data analytics system implementation environment. Inf. Process. Manage. 54(1), 791-806 (2018)

13. Espinosa, A.J., Armour, F.: The big data analytics gold rush: a research framework for coordination and governance. In: 49th Hawaii International Conference, Hawaii (2016) 
14. CGMA: From insight to impact - unlocking opportunities in big data. CGMA, New York (2013)

15. Dehinbo, J.: Theoretical base for developing a holistic knowledge management strategy for effective learning in organisations, Pretoria (2012)

16. Segarra, L., et al.: A framework for boosting revenue incorporating big data. J. Innov. Manage. 4(1), 39-68 (2016)

17. LaValle, S., Lesser, E., Shockley, R., Hopkins, M., Kruschwitz, N.: Big data, analytics and the path from insights to value. MIT Sloan Manage. Rev. 52(2), 21-32 (2011)

18. Peppard, J.: A tool to map your next digital initiative. Harvard Business Review, Berlin (2016)

19. Daniel, E., Peppard, J., Ward, J.: Managing the realization of business benefits from IT investments. MIS Q. Exec. 6(1), 1-11 (2007)

20. Humphrey, A.: SWOT analysis for management consulting. SRI Alumni Newsl. 1, 7-8 (2005)

21. Kaplan, R.S., Norton, D.P.: The balanced scorecard: translating strategy into action, Massachusetts (1996)

22. Schwalbe, K.: Information Technology Project Management, 7th edn. Cengage Technology Cengage Learning, Boston (2014)

23. Ward, J., Taylor, P., Bond, P.: Evaluation and realization of IS/IT benefits: an empirical study of current practice. Eur. J. Inf. Syst. 4(1), 214-225 (1996)

24. Wilson, H., Clark, M., Smith, B.: Justifying CRM projects in a business-to-business context: the potential of the benefits dependency network. Ind. Mark. Manage. 36(1), 770-783 (2007)

25. Ward, J., Daniel, E.: The strategic performance management cycle. Performance Measurement Association (2006)

26. Peppard, J., Ward, J., Daniel, E.: Managing the realization of business benefits from IT investments. MIS Q. Exec. 6(1), Article 3 (2008). https://aisel.aisnet.org/misqe/vol6/iss1/3

27. Ashraf, T.: Organisational development and big data: factors that impact successful big data implementations. ProQuest, Lisle (2017)

28. Leavitt, H., Bahrami, H.: Managerial Psychology: Managing Behavior in Organisations, 5th edn. University of Chicago, Chicago (1988)

29. Halaweh, M., Massry, A.E.: Conceptual model for successful implementation of big data in organisations. J. Int. Technol. Inform. Manage. 24(2), 21-34 (2015) 